\title{
Les symbioses dynamiques, mode de vie bactérien
}

\begin{abstract}
Le monde bactérien fonctionne à l'aide de symbioses faciles à improviser et adaptables. Leurs éléments de base : gènes et cellules sont interchangeables. La pression sélective constante du milieu actualise ou modifie les innombrables possibilités de ces symbioses dynamiques et efficaces, suivant les circonstances. Les bactéries, incluant les pathogènes, n'agissent donc jamais de façon isolée dans la nature. Selon le professeur S. Sonea, il n'y aurait donc pas de véritable espèce bactérienne.
\end{abstract}

\section{TIRÉS A PART}

S. Sonea : département de microbiologie et immunologie, faculté de médecine, pavillon principal, université de Montréal, CP 6128,

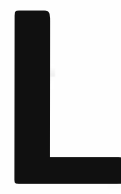

'enseignement de la microbiologie se fait souvent sans recours à un concept cohérent du monde bactérien. Les manuels décrivent bien les principaux mécanismes de transfert des gènes entre bactéries différentes mais ils ne s'attardent pas sur leurs conséquences décisives pour l'organisation du monde bactérien. La plupart de ces échanges génétiques sont accomplis par des gènes groupés dans des petites molécules circulaires d'ADN, contenant moins de un pour cent du génome cellulaire. On les appelle des petits réplicons parce qu'ils peuvent se reproduire de façon autonome dans un grand nombre de souches différentes. Il y en a deux catégories: les plasmides et les prophages. Quand ils sont présents dans une bactérie, ils lui font exprimer leurs gènes, comme s'ils faisaient partie du génome bactérien permanent. La concurrence vive qui règne entre les souches amplifie, par une multiplication cellulaire plus forte, la présence de ces gènes «visiteurs » s'ils amènent avec eux des caractéristiques favorables à l'adaptation à l'environnement. Cette sélection élimine (par «guérison») les prophages ou les plasmides inutiles ou nocifs. Comme ces petits réplicons constituent des centres génétiques supplémentaires dans la cellule qu'ils «visitent» ils représentent en fait une forme élémentaire d'endosymbiose [1].

\section{Le marché commun des gènes bactériens}

Une telle bactérie «infectée » pourrait, aussi, être considérée comme une «chimère », terme qui correspond à un organisme constitué d'un mélange de cellules provenant d'au moins deux espèces différentes ou de cellules qui contiennent au moins deux éléments génétiques de provenance différente. Les bactéries ont accès à un grand nombre de prophages et de plasmides qui, dans leur transfert d'une souche à l'autre, peuvent aussi entraîner d'autres gènes plus stables provenant de l'hôte précédent (figure 1, p. 379). A partir de cette riche «librairie» de gènes disponibles dans la nature, est constitué un véritable marché commun des gènes, manipulé par la sélection naturelle qui favorise les combinaisons les mieux adaptées aux circonstances immédiates. Non seulement les bactéries peuvent ainsi s'adapter mais elles peuvent aussi faire face à des problèmes pressants et les résoudre rapidement, surtout si la crise touche d'une façon durable toute une population de souches. Par exemple, nous savons que, menacés d'extermination par les antibiotiques, la plupart des bactéries pathogènes ont fini par recevoir, surtout à partir des bac- 


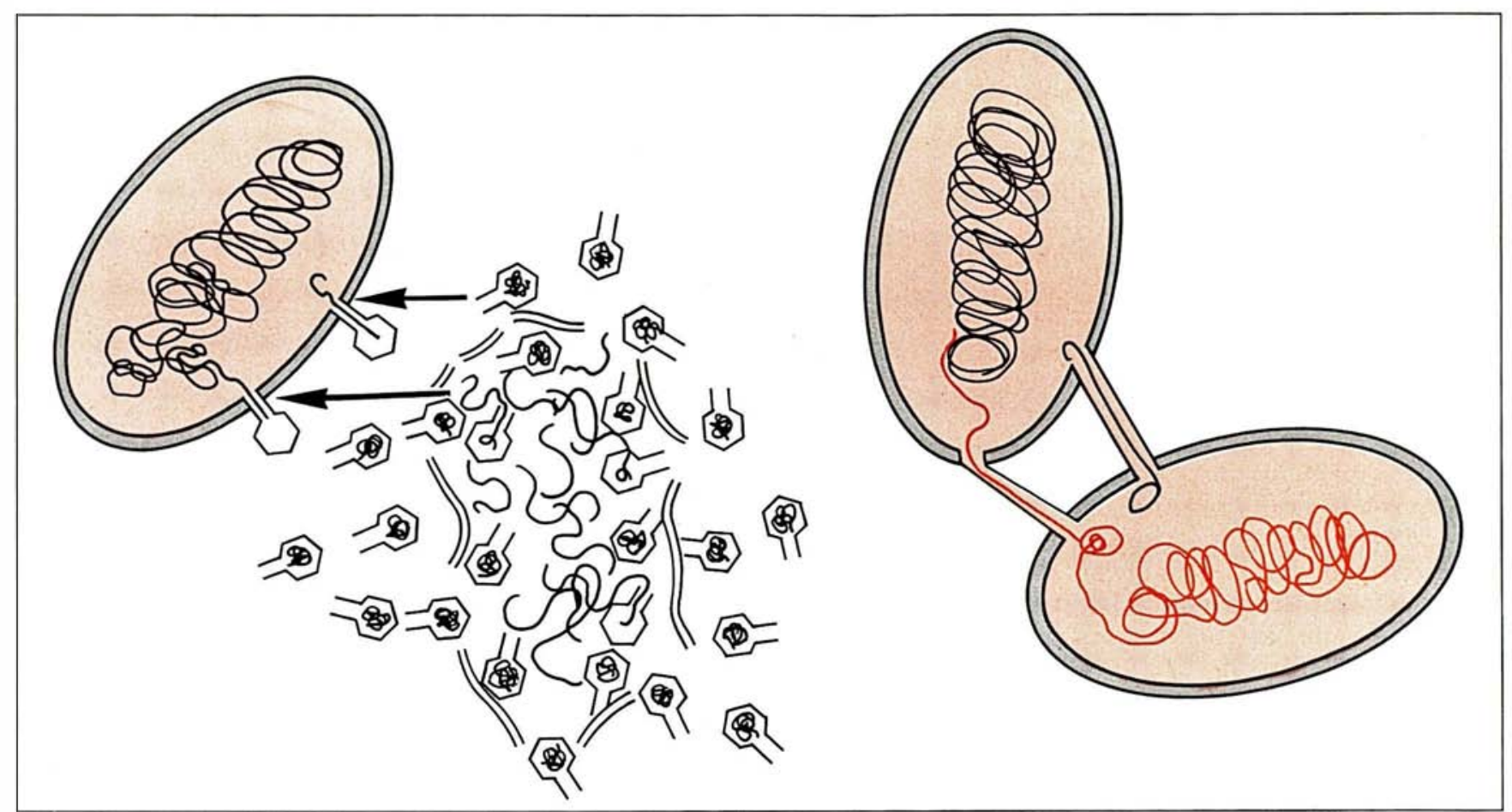

Figure 1. Plasmides de conjugaisons et prophages qui peuvent transférer activement leurs génomes et aussi des gènes appartenant à d'autres réplicons (transduction ou mobilisation). Quand les gènes proviennent du "chromosome » de la cellule donneuse, ils doivent s'aligner le long d'une zone d'homologie du "chromosome »de la cellule réceptrice et peuvent la remplacer.

téries du sol, des gènes de résistance à ces médicaments. Contrairement aux attentes initiales, les mutations n'ont pas joué un rôle important dans l'acquisition de cette résistance [2]. De la même façon, les substances biologiques utilisées en agriculture (insecticides, substances fertilisantes, etc.) et qui ont des propriétés antibactériennes, ont provoqué le déclenchement du même mécanisme de protection [3]. Dans la nature on ne trouve donc pas de lignée «pure» de bactérie. Toutes ont au moins un plasmide et un tiers des souches possèdent un ou plusieurs types de prophages. Tous ces éléments contribuent à titre temporaire, au génome bactérien. La variété de l'ensemble bactérien s'enrichit donc grâce à cette capacité d'acquisition des gènes provenant d'autres souches comme partie intégrante ou «à l'essai » dans leur génome, avec des possibilités de permutations et recombinaisons innombrables. Il en résulte une symbiose dynamique.
L'adaptabilité des cellules bactériennes est decefait considérablement accrue et le danger d'extinction par sur-spécialisation est fortement diminué [4].

\section{Des associations bactériennes pluricellulaires}

Ce mode de vie typiquement bactérien n'est pas limité au seul niveau unicellulaire. Nous le rencontrons aussi au niveau des associations bactériennes pluricellulaires. Celles-ci représentent l'équivalent biologique d'une plante ou d'un animal. Il s'agit de communautés formées par mélange, sans ordre apparent, de bactéries appartenant à des souches dont le métabolisme complémentaire permet une excellente répartition du travail d'adaptation. C'est le cas des populations bactériennes du sol, du rumen, etc. En réalité, l'immense majorité des souches bactériennes de la nature appartiennent à de telles entités [3, 4]. De façon analogue aux cellules morphologiquement et métaboliquement différenciées d'un être pluricellulaire, végétal ou animal, ces entités pluricellulaires bactériennes réunissent de nombreuses souches différentes sélectionnées par la compétition bactérienne permanente, émergeant des innombrables types de bactéries disponibles dans la biosphère. La majorité des souches trouvées dans de telles entités ne peuvent survivre en culture pure: elles ont besoin les unes des autres! D'un point de vue pratique, il n'y a dans la nature aucune souche bactérienne entièrement isolée et indépendante des cellules d'un type différent. Même quand une bactérie pathogène envahit un animal ou une plante, elle change d'environnement et devient un parasite des cellules eucaryotes ou de leurs produits. Elle est donc passée d'un type de dépendance cellulaire à un autre. 
Nous proposons donc l'hypothèse que la stratégie de la vie chez les bactéries est basée sur des associations faciles à réaliser et à modifier, utilisant un potentiel de cellules et de gènes qui souvent ne soutiendraient pas une vie indépendante dans la nature. Ces éléments fonctionnent comme des éléments de base interchangeables, pré-existants et largement disponibles, des véritables blocs de construction. La composition de leur mélange est sélectionnée en permanence (qualitativement et quantitativement) par la concurrence vive, toujours présente dans le monde bactérien. Nous avons précédemment décrit l'existence du génome potentiel commun [4] permettant à toute bactérie un accès aux gènes d'autres souches (figure 2). Il faut maintenant accepter également l'existence d'un «marché commun » de toutes les souches bactériennes de la planète. Elles possèdent une grande capacité à l'association en ectosymbiose.

\section{Une organisation solidaire}

Ces éléments de base et leurs activités évoquent un véritable ordinateur biologique. Il y a, d'une part, une banque («bibliothèque ») d'information, formée des gènes de toutes les bactéries, organisés d'habitude dans des sous-unités capables de se multiplier: petits réplicons ou cellules. L'ensemble mondial représente une variété métabolique qui dépasse de loin celle de tous les eucaryotes. La multiplication sélective des éléments de base dans des symbioses bien adaptées correspond aux possibilités d'amplification d'un ordinateur. Les divers choix sont déterminés par la concurrence permanente entre bactéries qui en est aussi le moteur. Nous proposons l'expression de «symbiose dynamique » pour décrire cette formule générale de vie dans le monde bactérien. On pourrait également parler de chimères dynamiques. L'aspect dynamique des symbioses est exclusif aux procaryotes. D'autre part, chez les eucaryotes, les symbioses ont une tendance à se figer habituellement sous forme de nouvelles espèces [5].

Le monde bactérien nous semble

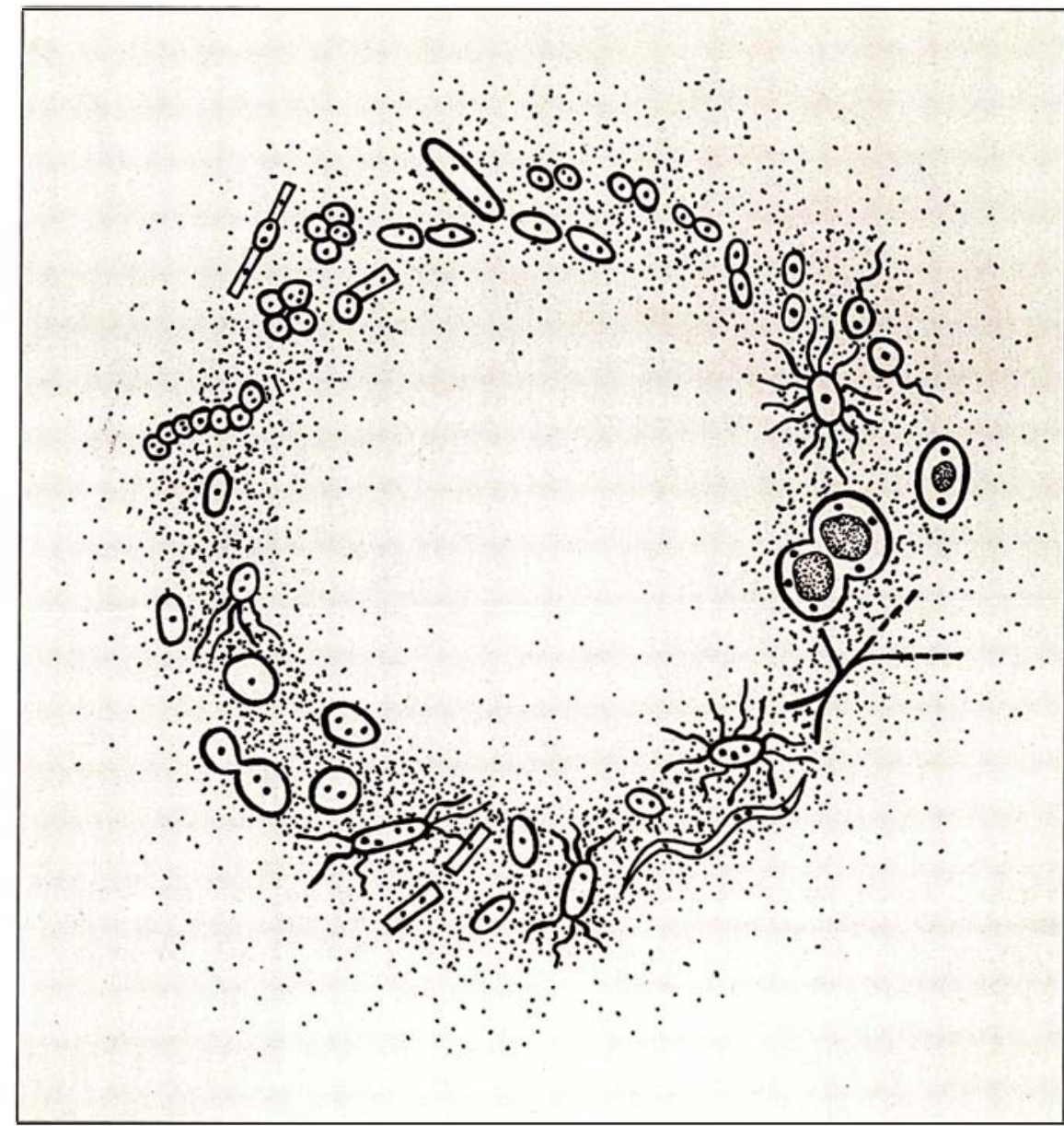

Figure 2. Représentation simplifiée du monde bactérien. La grande variété métabolique des cellules bactériennes est indiquée ici par des morphologies différentes. Les points représentent des petits réplicons, plasmides ou prophages. Une bonne partie de ces derniers sont disponibles à l'extérieur des cellules. II y en a au moins un dans chaque bactérie.

solidaire [2] de par la disponibilité générale de ses cellules et gènes différents. Ce concept cohérent et simple explique la façon de vivre des bactéries et s'applique aux divers niveaux de complexité de leurs activités. Cette réalité est fondamentalement différente de celle des eucaryotes et par conséquent essentielle pour la définition du monde bactérien.

A la lumière de cette hypothèse il conviendra de réexaminer deux chapitres importants de la bactériologie. - La classification générale des êtres vivants semble clairement commencer avec deux branches principales de l'arbre de la vie: (a) les bactéries ou procaryotes, incluant les Archaebac- téries qui possèdent également des petits réplicons [7, 8] et (b) les eucaryotes. Les premiers vivent organisés en symbioses dynamiques d'une grande souplesse qui annulent la notion «d'étranger » quand on parle d'autres cellules ou d'autres gènes. Il n'y a donc pas d'espèces véritables chez les bactéries.

- L'évolution chez les bactéries semble avoir favorisé la formation d'unités de base (gènes et cellules) très compétitives et sur-spécialisées, capables et obligées de s'associer et de se remplacer facilement. Isolées, elles restent incomplètes. L'évolution de tout le monde bactérien s'est probablement faite au bénéfice de l'ensemble. Ainsi tout nouveau gène devient 
disponible pour le patrimoine commun [4]. Cellules et petits réplicons ont connu une co-évolution entraînant une forte influence réciproque [1]. Le tout a fini par favoriser finalement l'accès généralisé au marché commun d'information héréditaire. L'évolution du monde bactérien s'est faite en perfectionnant un ensemble d'éléments de base communs, capables et obligés de s'associer dans d'innombrables symbioses temporaires, faciles à improviser et à s'adapter. Des épisodes évolutifs reversibles et temporaires se font en permanence dans le monde bactérien

\section{S. Sonea}

Professeur à l'université de Montréal. Département de microbiologie et immunologie, faculté de médecine, pavillon principal, Université de Montréal, CP 6128, Succ. A, Montréal, Québec, H3C 3J7, Canada.

\section{RÉFÉRENCES}

1. Sonea S. Bacterial viruses, prophages and plasmids, reconsidered. Ann NY Acad Sci 1987 ; 503 : $251-60$.

2. Sonea S, Panisset M. L'évolution des infections bactériennes et la solidarité génétique des bactéries. Med Hyg 1978; 36 : 2074-81.

3. Bull AT, Slater JH. Microbial interactions and communities. Londres: Academic Press, 1982

4. Sonea S, Panisset M. A new bacteriology Boston: Jones and Bartlett, 1983.

5. Margulis L. Symbiosis in cell evolution. San Francisco: W.A. Freeman, 1981

6. Sonea S. A tentative unifying view of bacteria. Rev Canad Biol 1971 ; 30 : 239-44

7. Zillig W, Yeats S, Holtz L, Bock A, Lutz S. Plasmid-related anaerobic autotrophy of the novel Archaebacterium, Sulfobolus ambivalens. Nature 1985 ; 313 : 789-91.

8. Zillig W, Grapp F, Henschen N, et al. Archaebacterial virus host systems. Syst Appl Microb 1986; 7 : 58-66.

$\mathrm{m} / \mathrm{s} n^{\circ} 6$ vol. 4 , juin 88

\section{BRÈVES}

Les récepteurs nucléaires peuvent inhiber ou activer la transcription par l'intermédiaire de domaines et selon des mécanismes différents. Les hormones en général, les stéroïdes en particulier, activent certains gènes et en inhibent d'autres. La question des mécanismes de l'inhibition transcriptionnelle par les hormones restait à ce jour entière. Un élément de réponse vient d'être donné sur le modèle du gène de prolactine par l'équipe de M.G. Rosenfeld (San Diego, la Jolla, Californie, USA) [1]. Ce gène est activé par les œestrogènes par l'intermédiaire de la liaison du complexe hormone/récepteur à un élément spécifique d'ADN. Il est inhibé par les glucocorticoïdes et par les œestrogènes euxmêmes en cas de mutation ou de délétion de l'élément d'ADN spécifique du récepteur des œestrogènes. Des expériences de mutation de la séquence codant pour le récepteur ont montré que la région $\mathrm{C}$ de liaison à l'ADN, essentielle à l'activation transcriptionnelle, n'intervenait pas dans l'inhibition transcriptionnelle induite par l'hormone. C'est la région charnière $\mathrm{D}$, située immédiatement à côté de la précédente, à l'extrémité $\mathrm{COOH}$-terminale, qui semble indispensable à l'inhibition. Un récepteur hybride, dont la charnière est remplacée par celle du récepteur des glucocorticoïdes, conserve son pouvoir inhibiteur lorsqu'il lie l'hormone, ce qui peut sembler surprenant compte tenu de l'absence de conservation de cette région dans les différents récepteurs nucléaires $\left(\mathrm{m} / \mathrm{s} n^{\circ} 3\right.$, vol. $3, p .172$ et $n^{\circ} 3$, vol. 4, p. 196). En fait, ces résultats évoquent ceux notés à propos des facteurs de transcription de levure GAL4 et GCN4 : la région protéique intervenant dans l'effet transcriptionnel peut être remplacée par des séquences variées sans que les facteurs perdent leur activité $\left(\mathrm{m} / \mathrm{s} n^{\circ} 6\right.$, vol. 4, p. 385). Une région du récepteur pratiquement limitée au peptide formant cette charnière se comporte comme un inhibiteur transcriptionnel indépendant des hormones.
L'inhibition exige la présence au niveau du promoteur de plusieurs éléments d'ADN qui sont eux-mêmes des sites de fixation d'autres facteurs de transcription. Il semble donc que l'inhibition nécessite l'interaction entre la région $\mathrm{D}$ du récepteur des stéroïdes (peut-être rendue accessible par la liaison de l'hormone à la région $\mathrm{E}$ ) avec d'autres protéines modulant la transcription du gène. D'autres exemples d'une telle modulation de l'activité de facteurs de transcription par leur interaction avec d'autres protéines sont maintenant parfaitement bien connus. Les résultats discutés ici démontrent ainsi que les récepteurs nucléaires peuvent contrôler la transcription de différentes manières, l'inhibition et l'activation transcriptionnelles faisant intervenir des domaines protéiques différents.

[1. Adler S, et al. Cell 1988; $52: 685$ 95.]

Le gène codant pour la chaîne $\beta_{2}$ du gène TCR ( $T$ cell receptor) possède un activateur situé loin en aval du gène. Les gènes des chaînes lourdes et de la chaîne légère $\kappa$ possèdent un enhancer situé dans l'intron séparant, après réarrangement dans les cellules $B$, les segments codant pour la partie variable et la partie constante. Un même type de enhancer vient d'être détecté à $5 \mathrm{kpb}$ en 3' du segment $C \beta 2$ codant pour la partie constante $\beta 2$ de la chaine $\beta$ du récepteur pour l'antigène des cellules $T$ [1]. Cet élément est indispensable à l'expression du gène $\beta$ dans des souris transgéniques. A noter que ce enhancer $\beta$ est à $18 \mathrm{kpb}$ en $3^{\prime}$ du promoteur du gène $\beta 2$ réarrangé... et semble être aussi utilisé en cas de réarrangement du gène $\beta 1$; dans ce dernier cas le enhancer est à $27 \mathrm{kpb}$ du promoteur! La logique de la présence d'un enhancer en aval du promoteur de gènes subissant un réarrangement en amont est évidente: il s'agit d'éviter une délétion de la séquence stimulatrice au cours du processus de réarrangement.

[1. Krimpenfort P, et al. Embo J $1988 ; 7: 745-50$.] 